\title{
ON THE ALGEBRAIC CONVERGENCE OF FINITELY GENERATED KLEINIAN GROUPS IN ALL DIMENSIONS
}

\author{
XI FU \\ (Received 10 May 2011)
}

\begin{abstract}
Let $\left\{G_{r, i}\right\}$ be a sequence of $r$-generator Kleinian groups acting on $\overline{\mathbb{R}}^{n}$. In this paper, we prove that if $\left\{G_{r, i}\right\}$ satisfies the $F$-condition, then its algebraic limit group $G_{r}$ is also a Kleinian group. The existence of a homomorphism from $G_{r}$ to $G_{r, i}$ is also proved. These are generalisations of all known corresponding results.
\end{abstract}

2010 Mathematics subject classification: primary 30F40; secondary 20H10, 57S30.

Keywords and phrases: Kleinian group, WY(G), loxodromic element, algebraic convergence.

\section{Introduction}

In this paper, we will adopt the same definitions and notation as in $[5,7,8]$, such as discrete groups $G$ of $M\left(\overline{\mathbb{R}}^{n}\right)$, limit sets $L(G)$ of $G$, nonelementariness and so on. For example, $G$ is a Kleinian group if $G$ is discrete and nonelementary.

Let $\left\{G_{r, i}\right\}$ be a sequence of subgroups in $M\left(\overline{\mathbb{R}}^{n}\right)$ and each $G_{r, i}$ be generated by $g_{1, i}, g_{2, i}, \ldots, g_{r, i}(0<r<\infty)$. If, for each $t \in\{1,2, \ldots, r\}$,

$$
g_{t, i} \rightarrow g_{t} \in M\left(\overline{\mathbb{R}}^{n}\right) \text { as } i \rightarrow \infty,
$$

then we say that $\left\{G_{r, i}\right\}$ converges algebraically to $G_{r}=\left\langle g_{1}, g_{2}, \ldots, g_{r}\right\rangle$ and $G_{r}$ is called the algebraic limit group of $\left\{G_{r, i}\right\}$. If, for each $i, G_{r, i}$ is a Kleinian group, then the question when $G_{r}$ is still a Kleinian group has attracted much attention. For example, in [3], Jørgensen and Klein established the following classical algebraic convergence theorem.

THEOREM A [3]. Let $\left\{G_{r, i}\right\}$ be a sequence of r-generator Kleinian groups of $M\left(\overline{\mathbb{R}}^{2}\right)$ converging algebraically to the group $G_{r}$. Then $G_{r}$ is a Kleinian group.

In higher dimensions, Martin observed that if the sequence $\left\{G_{r, i}\right\}$ contains elliptic elements $g_{t, i}$ such that $g_{t, i} \rightarrow g_{t}$ with ord $\left(g_{t, i}\right) \rightarrow \infty$ as $i \rightarrow \infty$, then the algebraic limit

The research was partly supported by NSF of China (No. 11071063).

(C) 2011 Australian Mathematical Publishing Association Inc. 0004-9727/2011 \$16.00 
group is not a Kleinian group, where 'ord $(g)$ ' denotes the order of $g$. This shows that to study when the algebraic limit group of a sequence of $r$-generator Kleinian groups is Kleinian some restriction is needed. In [5], Martin introduced the following restriction.

A set $X$ of $M\left(\overline{\mathbb{R}}^{n}\right)$ is said to have uniformly bounded torsion if there is an integer $N>0$ such that for each $g \in X$,

$$
\operatorname{ord}(g) \leq N \quad \text { or } \quad \operatorname{ord}(g)=\infty \text {. }
$$

By using this restriction, Martin generalised Theorem A to the higher dimensional case.

Theorem B [5, Proposition 5.8]. Let $G_{r}$ be the algebraic limit group of a sequence $\left\{G_{r, i}\right\}$ of r-generator Kleinian groups of $M\left(\overline{\mathbb{R}}^{n}\right)$ with uniformly bounded torsion. Then $G_{r}$ is a Kleinian group.

Recently, Wang [7] and Yang [10] introduced the restrictions 'EP-condition' and 'Condition A', respectively, to weaken 'uniformly bounded torsion'. Their results are as follows.

Theorem C [7, Theorem 1.1]. Let $G_{r}$ be the algebraic limit group of a sequence $\left\{G_{r, i}\right\}$ of r-generator Kleinian groups of $M\left(\overline{\mathbb{R}}^{n}\right)$. If $\left\{G_{r, i}\right\}$ satisfies the EP-condition, then $G_{r}$ is a Kleinian group.

Here a sequence $\left\{G_{i}\right\}$ is said to satisfy the EP-condition if the following two conditions are satisfied.

(1) For any sequence $\left\{f_{i k}\right\}, f_{i k} \in G_{i k}\left(\in\left\{G_{i}\right\}\right)$, if $\operatorname{card}\left(\operatorname{fix}\left(f_{i k}\right)\right)=\infty$ and $f_{i k} \rightarrow f$ as $k \rightarrow \infty$, where $f$ is the identity map $I$ or a parabolic element, then $\left\{f_{i k}\right\}$ has uniformly bounded torsion.

(2) $\left\{G_{i}\right\}$ satisfies Property $A$, that is, $\left\{G_{i}\right\}$ contains no sequences $\left\{f_{i k}\right\},\left\{g_{i k}\right\}$ which satisfy that both $f_{i k}, g_{i k} \in G_{i k}\left(\in\left\{G_{i}\right\}\right)$ are elliptic and

$$
\begin{gathered}
\operatorname{fix}\left(f_{i k}\right) \cap \operatorname{fix}\left(g_{i k}\right)=\emptyset, \quad \operatorname{card}\left(\operatorname{fix}\left(f_{i k}\right)\right)=\operatorname{card}\left(\operatorname{fix}\left(g_{i k}\right)\right)=2, \\
f_{i k} \rightarrow I \quad \text { and } \quad g_{i k} \rightarrow I
\end{gathered}
$$

as $k \rightarrow \infty$.

THEOREM D [10, Theorem 2.4]. Let $G_{r}$ be the algebraic limit group of a sequence $\left\{G_{r, i}\right\}$ of r-generator Kleinian groups of $M\left(\overline{\mathbb{R}}^{n}\right)$. If $\left\{G_{r, i}\right\}$ satisfies Condition $A$, then $G_{r}$ is a Kleinian group.

Here we say that a sequence $\left\{G_{i}\right\}$ satisfies Condition $A$ if there is no sequence $\left\{f_{i k}\right\}, f_{i k} \in G_{i k}\left(\in\left\{G_{i}\right\}\right)$ with $\operatorname{card}\left(\operatorname{fix}\left(f_{i k}\right)\right)=\infty$ and $f_{i k} \rightarrow I$ as $k \rightarrow \infty$ (see [2]).

ExAmple 1.1. Suppose that $G_{2}=\left\langle f_{1}, f_{2}\right\rangle$ is a two-generator purely hyperbolic nonelementary subgroup of $\operatorname{PSL}(2, \mathbb{R})$ and that, for each natural number $i$,

$$
f_{i}=\left(\begin{array}{cc}
a_{i} & 0 \\
0 & a_{i}
\end{array}\right),
$$


where $a_{i}=\cos \left(\theta_{i} \pi\right)+e_{2} e_{3} \sin \left(\theta_{i} \pi\right)$ and each $\theta_{i}$ is a rational number. Let

$$
G_{2, i}=\left\langle G_{2}, f_{i}\right\rangle
$$

Then, for each $i, G_{2, i}$ is a Kleinian group in $\operatorname{PSL}\left(2, \Gamma_{4}\right)$. If the sequence $\left\{\theta_{i}\right\}$ converges to a rational number $\theta$, then the algebraic limit group $G_{3}$ of $\left\{G_{2, i}\right\}$ is also a Kleinian group; but, if the sequence $\left\{\theta_{i}\right\}$ converges to an irrational number $\theta$, then $G_{3}$ is nondiscrete. Moreover, in the former case, if $\theta_{i}=1 / 3^{i}$, then we know that the sequence $\left\{G_{2, i}\right\}$ does not satisfy the $E P$-condition nor Condition $A$, but $G_{3}$ is still a Kleinian group.

Motivated by Example 1.1, we introduce the following restriction.

Definition 1.2. We say that a sequence $\left\{G_{i}\right\}$ satisfies the $F$-condition if there is no sequence $\left\{f_{i k}\right\}, f_{i k} \in W Y\left(G_{i k}\right)\left(\in\left\{G_{i}\right\}\right)$ such that $f_{i k} \rightarrow f$ as $k \rightarrow \infty$, where $f$ is an elliptic element with ord $(f)=\infty$.

Let us recall the important notation $W Y(G)$ for a Kleinian group $G$, which was first put forward by Wang and Yang in [8]:

$$
W Y(G)=\left\{f:\left.f\right|_{M(G)}=I, f \in G\right\},
$$

where $M(G)$ is the smallest $G$-invariant hyperbolic space whose boundary contains the limit set $L(G)$ of $G$ (see [6]). It is obvious that $W Y(G)$ is $\{I\}$ or a purely elliptic subgroup of $G$.

REMARK 1.3. Obviously, if a sequence of Kleinian groups satisfies the EP-condition or Condition $A$, then it must satisfy the $F$-condition. From Example 1.1, we see that there are sequences of Kleinian groups which satisfy the $F$-condition but do not satisfy the $E P$-condition nor Condition $A$. Also, if a sequence $\left\{G_{r, i}\right\}\left(\left\{W Y\left(G_{r, i}\right)\right\}\right)$ of Kleinian groups has uniformly bounded torsion, then $\left\{G_{r, i}\right\}$ satisfies the $F$-condition.

By using the $F$-condition, we get the following generalisation of Theorems B, C and D.

THEOREM 1.4. Let $G_{r}$ be the algebraic limit group of a sequence $\left\{G_{r, i}\right\}$ of r-generator Kleinian groups of $M\left(\overline{\mathbb{R}}^{n}\right)$. If $\left\{G_{r, i}\right\}$ satisfies the $F$-condition, then $G_{r}$ is a Kleinian group.

We have the following corollary, which is easily derived from Theorem 1.4 and Remark 1.3.

Corollary 1.5. Let $G_{r}$ be the algebraic limit group of a sequence $\left\{G_{r, i}\right\}$ of r-generator Kleinian groups of $M\left(\overline{\mathbb{R}}^{n}\right)$. If $\left\{W Y\left(G_{r, i}\right)\right\}$ has uniformly bounded torsion, then $G_{r}$ is a Kleinian group.

Moreover, we prove the following result, which is a generalisation of [5, Theorem 6.1]. 
THeORem 1.6. Let $\left\{G_{r, i}\right\}$ be a sequence of r-generator Kleinian groups of $M\left(\overline{\mathbb{R}}^{n}\right)$ converging algebraically to the group $G_{r}$. Suppose that the corresponding sequence $\left\{W Y\left(G_{r, i}\right)\right\}$ of $\left\{G_{r, i}\right\}$ has uniformly bounded torsion and that $G_{r}$ is finitely presented. Then $G_{r}$ is also a Kleinian group and the correspondence from the generators of $G_{r}$ to their approximants in $G_{r, i}$ extends for all sufficiently large $i$ to a homomorphism of $G_{r}$ onto $G_{r, i}$.

\section{Proofs of Theorems 1.4 and 1.6}

2.1. Several lemmas. The following result due to Waterman is from [9].

Lemma $\mathrm{E}$ [9, Theorem 11]. If $\langle f, g\rangle$ is a Kleinian group of $M\left(\overline{\mathbb{R}}^{n}\right)$, then

$$
\|f-I\| \cdot\|g-I\|>\frac{1}{32} \text {. }
$$

The following two lemmas are crucial for the proofs of Theorems 1.4 and 1.6.

Lemma 2.1. Let $G_{r}$ be the algebraic limit group of a sequence $\left\{G_{r, i}\right\}$ of $r$-generator Kleinian groups of $M\left(\overline{\mathbb{R}}^{n}\right)$. Then:

(1) $G_{r}$ is nonelementary; and

(2) $G_{r}$ is nondiscrete if and only if there exists an elliptic element $f \in W Y\left(G_{r}\right)$ with $\operatorname{ord}(f)=\infty$.

Proof. The first part of this lemma follows from [4, Theorem 1.4]. Now we come to prove the second part. It suffices to show that if $G_{r}$ is nondiscrete, then there is an element $f \in W Y\left(G_{r}\right)$ with ord $(f)=\infty$, since the converse is obvious. Now we assume that $G_{r}$ is nondiscrete. Recall that $G_{r}$ is a finitely generated subgroup of $M\left(\overline{\mathbb{R}}^{n}\right)$. By applying the Selberg lemma, we know that $G_{r}$ contains a torsion free subgroup $G_{r}^{\prime}$ of finite index which is nondiscrete as well. Then there exists a sequence $\left\{f_{j}\right\}$ in $G_{r}^{\prime}$ such that $f_{j} \rightarrow I$ as $j \rightarrow \infty$. As $G_{r}^{\prime}$ is nonelementary, there are finitely many loxodromic elements $g_{1}, g_{2}, \ldots, g_{s}$ in $G_{r}^{\prime}$ such that the set $\left\{\operatorname{fix}\left(g_{1}\right), \operatorname{fix}\left(g_{2}\right), \ldots, f i x\left(g_{s}\right)\right\}$ spans the boundary of $M\left(G_{r}^{\prime}\right)$. Then, for all sufficiently large $j$, we have

$$
\left\|f_{j}-I\right\| \cdot\left\|g_{k}-I\right\|<\frac{1}{32},
$$

where $k \in\{1,2, \ldots, s\}$. Let $f_{i, j}$ and $g_{i, k}$ be the corresponding elements of $f_{j}$ and $g_{k}$ in $G_{r, i}$, respectively. Then, for large enough $i$,

$$
\left\|f_{i, j}-I\right\| \cdot\left\|g_{i, k}-I\right\|<\frac{1}{32} .
$$

Lemma $\mathrm{E}$ implies that the subgroups $\left\langle f_{i, j}, g_{i, k}\right\rangle$ are elementary. It follows that fix $\left(g_{i, k}\right) \subset \operatorname{fix}\left(f_{i, j}\right)$, which shows that for $k \in\{1,2, \ldots, s\}$ and all sufficiently large $j$, fix $\left(g_{k}\right) \subset \operatorname{fix}\left(f_{j}\right)$. Hence, $f_{j} \in W Y\left(G_{r}^{\prime}\right)$, from which the conclusion follows.

Lemma 2.2. Let $\left\{G_{i}\right\}$ be a sequence of finitely generated Kleinian groups of $M\left(\overline{\mathbb{R}}^{n}\right)$ converging algebraically to a group $G$. If there exists a sequence $\left\{f_{i k}\right\}, f_{i k} \in G_{i k}$ $\left(\in\left\{G_{i}\right\}\right)$, such that $f_{i k} \rightarrow I$ as $k \rightarrow \infty$, then, for sufficiently large $k, f_{i k} \in W Y\left(G_{i k}\right)$. 
Proof. By [4, Lemma 4.2], we know that for large enough $k, f_{i k}=I$ or there is a $G_{i k}$-invariant hyperbolic space $\Pi_{i k}$ which is fixed pointwise by $f_{i k}$. So, the closed set $\bar{\Pi}_{i k} \cap \overline{\mathbb{R}}^{n}$ is also $G_{i k}$-invariant. Since the limit set $L\left(G_{i k}\right)$ of $G_{i k}$ is the smallest $G_{i k}$-invariant subset in $\overline{\mathbb{R}}^{n}$, similar reasoning as in [1, Theorem 5.3.7] shows that $L\left(G_{i k}\right) \subset \bar{\Pi}_{i k} \cap \overline{\mathbb{R}}^{n}$, which implies that $M\left(G_{i k}\right) \subset \Pi_{i k}$. It follows that $f_{i k} \in W Y\left(G_{i k}\right)$.

2.2. Proof of Theorem 1.4. By Lemma 2.1, we only need to prove that there is no elliptic element $f \in W Y\left(G_{r}\right)$ with ord $(f)=\infty$. Suppose on the contrary that there is some elliptic element $f \in W Y\left(G_{r}\right)$ such that $\operatorname{ord}(f)=\infty$. Then there exists an integer sequence $\left\{n_{j}\right\}$ such that $f^{n_{j}} \rightarrow I$ as $n_{j} \rightarrow \infty$. For each $n_{j}$, let $f_{i}^{n_{j}}$ be the corresponding element in $G_{r, i}$. By Lemma 2.2 and the hypothesis that $\left\{G_{r, i}\right\}$ satisfies the $F$-condition, we know that $f_{i}^{n_{j}}=I$ for large enough $i$. It follows that $f^{n_{j}}=I$, which contradicts the assumption that $f \in W Y\left(G_{r}\right)$ with $\operatorname{ord}(f)=\infty$.

2.3. Proof of Theorem 1.6. The proof easily follows from Lemma 2.2 and a similar argument as in the proof of [5, Theorem 6.1].

\section{Acknowledgement}

The author would like to thank the referee for a careful reading of this paper as well as for many useful comments and suggestions.

\section{References}

[1] A. F. Beardon, The Geometry of Discrete Groups, Graduate Texts in Mathematics, 91 (Springer, New York, 1983).

[2] A. Fang and B. Nai, 'On the discreteness and convergence in $n$-dimensional Möbius groups', J. Lond. Math. Soc. 61 (2000), 761-773.

[3] T. Jørgensen and P. Klein, 'Algebraic convergence of finitely generated Kleinian groups', $Q . J$. Math. Oxford 33 (1982), 325-332.

[4] M. Kapovich, 'On the sequences of finitely generated discrete groups, in the tradition of AhlforsBers. V', Contemp. Math. 510 (2010), 165-184.

[5] G. Martin, 'On discrete Möbius groups in all dimensions: a generalization of Jørgensen's inequality', Acta Math. 163 (1989), 253-289.

[6] X. Wang, 'Dense subgroups of $n$-dimensional Möbius groups', Math. Z. 243 (2003), 643-651.

[7] X. Wang, 'Algebraic convergence theorems of $n$-dimensional Kleinian groups', Israel J. Math. 162 (2007), 221-233.

[8] X. Wang and W. Yang, 'Discreteness criteria of Möbius groups of high dimensions and convergence theorems of Kleinian groups', Adv. Math. 159 (2001), 68-82.

[9] P. Waterman, 'Möbius transformations in several dimensions', Adv. Math. 101 (1993), 87-113.

[10] S. Yang, 'Algebraic convergence of finitely generated Kleinian groups in all dimensions', Linear Algebra Appl. 432 (2010), 1147-1151.

XI FU, Department of Mathematics, Shaoxing College of Arts and Sciences, Shaoxing, Zhejiang 312000, PR China

e-mail: fuxi1000@yahoo.com.cn 